\title{
Evaluation of Methods of Volume-Production of Vodcasts of Presentations
}

\author{
W.A.McKee, D.K.Harrison and M.Allan \\ Glasgow Caledonian University, Scotland
}

\begin{abstract}
This paper describes traditional methods of production of videos of lecture presentations and argues that these are suitable only for a limited number of presentations due to the high cost of capture and postproduction. The concept of making every presentation of every class available as a podcast is discussed, and methods of utilising low-cost technologies and applications to allow this to be achieved are compared. Experiences of pilot schemes utilising a variety of devices and commercial, freeware and self-written production methods will be presented, with comment on the usability of each approach at both capture and production stages.
\end{abstract}

Index Terms-Lecture capture, Podcasts, Student-centred learning, Blended learning

\section{INTRODUCTION}

There has been a long-running debate on the role of the basic presentation or lecture in modern education. (Gibbs, 1992) states that "the dominance of lecturing in higher has little rational justification". There were strong arguments to dispose of "expository teaching" in favour of "discovery learning" in the 1960s, Shulman and Keisal, 1966), from which grew a push to student-centred learning heavily dependent on "Educational technology" (Rowntree, 1992). However it is clear that lectures and presentations from a "knowledgeable" person are still highly popular with both providers and consumers of education - whatever the reasons. What is clear however is that the concept of being there in person is not viewed as an attractive proposition to many who wish to experience the presentation. In the professional sphere there are simply too many difficulties involved in getting large numbers of people to one place - unless that is a "conference" in Las Vegas or some similar enticing location. If the personage is august enough, there are physical problems in finding a venue large enough for the audience. On a more prosaic note, attendances at all kinds of timetabled events in educational establishments are a particular concern - even those students who are committed to the course do not feel it is important to attend at the levels of a generation before.

This has led to the rapid growth of "pre-recorded" media content. There have been a number of courses where the "video lecture" has been a core element of the teaching methodology, notably the materials produced by the Open University in the UK. In the professional world, many companies have produced material for educational purposes (Cisco, 2008) or fro corporate information (SUN, 2008), or to make the keynote speeches by august persons available to the masses, such as the talks by industry leaders to institutions (IEE, 2008). Commercial equivalents to the specialist publishing houses have also sprung up to cater for niche presentations in engineering, legal or medical fields (Boxmind, 2008).

With the advent of mobile technologies with suitably large bandwidths, it is now possible to view this recorded media almost anytime, anywhere - as a "video podcast". Thus there is a demand for every presentation which a student may have not attended to be available for their "offline" perusal, and an unwillingness of the consumer to accept that the institution should not make it possible for the staff to achieve this.

From the academic's point of view, there has been both enthusiasm and concern. There are some clear opportunities for improving the understanding and retention - from the basic fact that the student actually gets to see the lecture they missed, to the capability of repetition and review of individual topics in the presentation impossible in anything other than one-to-one situations. The lecturer can also use the video as a basis for a range of associated material, through tutorials, supporting media and online resources, all the way to direct links to the mechanisms available in online learning environments (MLE's) such as (Blackboard, 2008). Concerns include the possiblility of an incitement to nonattendance, but studies have shown that this does not actually have a significant effect (Philips, 2007), with those who were inclined to attend still doing so, and those who were not so inclined having been recorded as viewing the material they would have otherwise have missed. There are also concerns about the "professional" look of the recorded presentation, but at a recent seminar in the author's University it was argued that the "U-Tube" generation were unconcerned about these niceties.

\section{LECTURE VIDEO CAPTURE TECHNIQUES.}

\section{A. "Studio quality".}

The authors have been involved with "off-site" or "work-based" learning for many years.(Rose and McKee, 2001). As part of that course it was necessary that lectures were to be available in multiple locations at multiple times - clearly not practicable for an individual. Due to security issues, many of the locations of the students were not compatible with "broadcast" lecture techniques such as the ISDN-based teleconferencing equipment then in use. The solution was therefore to record the lectures to videotape.

In order to do this, the Audio-Visual support services of the University were involved. These were professionals and the lecturers were surprised by the sheer scale of the 
process involved in producing "studio quality" video. To begin with the venue had to be suitable both in "sightlines" and for audio - and considering these were purpose built lecture theatres it was a revelation how badly many of them were considered "unsuitable". Then there were the personnel - two camera operators, a sound person, and a "gofer" who set up the lighting rig and timed the events of the presentation. Then the actual recording was required to be redone several times, so that there would be no need for "retakes" at al later date. Thus a one-hour lecture took a full day to capture. After capture the AV post-production team used a professional editing suite to produce a "clean" version of the lecturer's performance from the several takes. They then used a "split-screen" hardware video facility to add the presentation materials (consisting of powerpoint slides, screenshots of applications, and stills of objects) to the presentation. This apparently took two days of the editor's time. The resulting video was then duplicated and distributed.

This effort was funded by the external income generated, however as can be imagined it was very costly (the only person who did not have to be paid was the lecturer!) and was clearly a "big deal" and thus is appropriate only in the most special cases. This is therefore not a suggested method fro producing podcasts on a wide scale.

\section{B. Capture Stations}

Many establishments have invested in adding video and audio capture equipment into specific auditoria. This permanent kit is usually professional quality and may well be remote controlled to allow for the most flexible capture of the presentation, using multiple cameras and microphones. Thus these are expensive to install and usually require at least one technical person to operate the basic capture. Often this is used to broadcast the presentation to similar "repeater" auditoria, giving the capability to extend the lecture in a geographical sense. For timeshifting however there are a few difficulties.

If there is only one operator, then they have to make onthe-spot decisions about which camera is "active". There has to be a decision about the "secondary channels" (eg the slideshow)- are they on a separate data channel, driving a second projector, or are they automatically (by hardware) embedded into the stream. This clearly makes a difference in the recording taken - multiple streams will usually need post processing as before.

The key point however is that of practicality. Although such installations are much cheaper than the "studio" system, there is still a need for an operator and most crucially the location is fixed and expensive - again it is not possible for every lecturer to record every session of their course in such a facility. So again a very useful method but NOT suitable for the wide-scale production of podcasts.

\section{Web-Based Lecture technologies (WBLT)}

There are a number of commercial organisations which have extended the early teleconferencing mechanisms and auditorium capture station concept to attempt to reduce the costs and need for extra technical help. These mechanisms (Lectopia, 2007) or (Apreso, 2007), (now both part of ECHO360, 2008) typically attempt to automate the operation of the process, and use specialised capture equipment and webserver technology to avoid the post-processing overhead.

The presenter is required to ensure the presentation material is connected to the capture station appropriately, although this may be as simple as plugging in a memory card or CD and "browsing" to it. Other systems allow direct connection of a laptop to the capture station in parallel with that to the projector. Microphones and/or audio sources must be connected, and usually have to be "set-up" for level. Some systems also allow for secondary cameras, rostrum (object) cameras, smartboards, and drawing tablets to be connected, though how these are integrated into the video stream is an extra task.
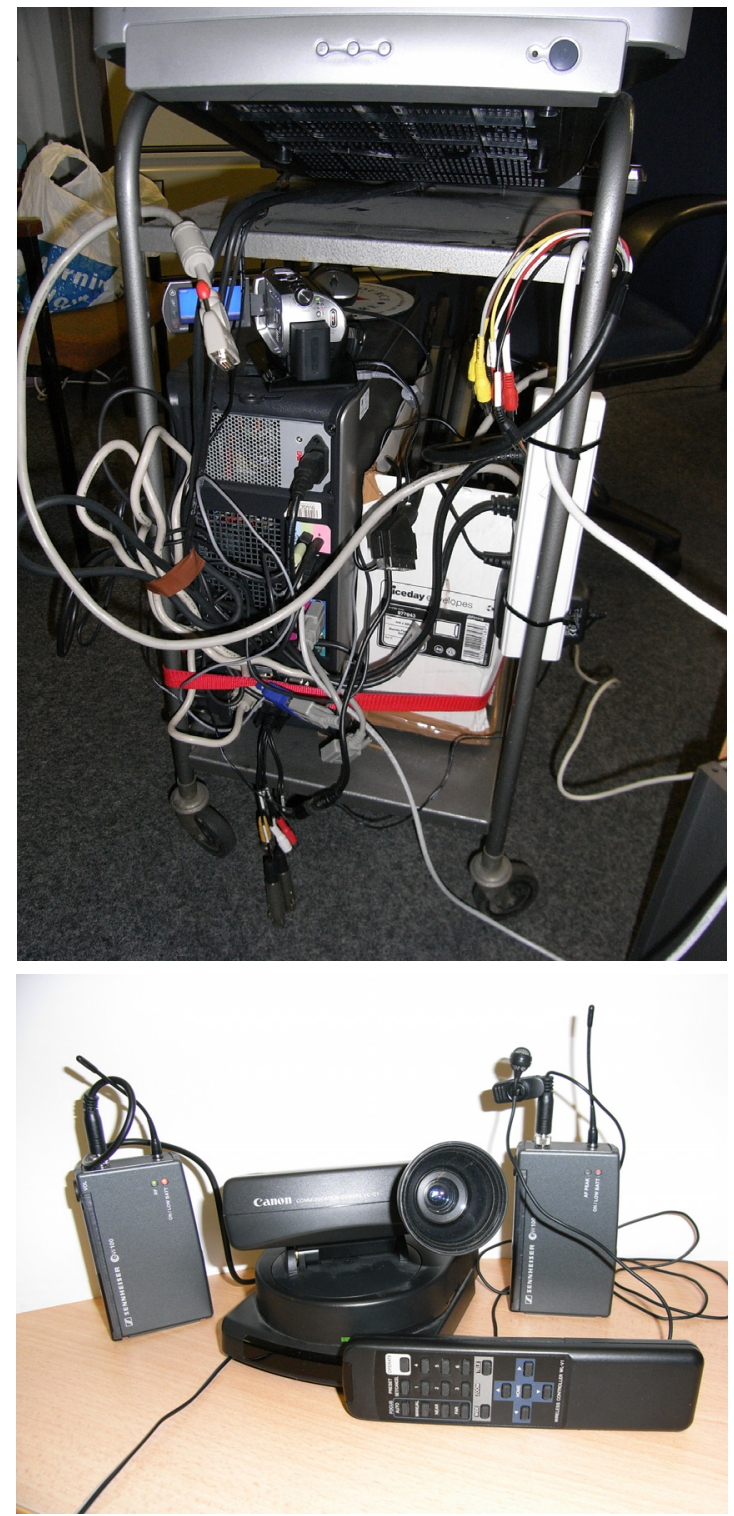

Figure 1. A typical capture station (note usually hidden in a cupboard), with a typical remote camera and radio microphone

This works well and is in use in many places. The key drawbacks are that again only a limited number of places are "wired" and demand for them is oversubscribed. Although management is less, there is still an operator cost hidden in the background management. However one of the key drawbacks is the costing model - although the equipment is bought outright, most of these operate on an annual "licence" basis, which can be too expensive for a 
department to consider, meaning the whole institute must be convinced to invest in such technologies.

The actual capture, post processing and storage of the podcast is controlled by a "script" set up in the WBLT management system, which defines the video integration and directs the output to the required places, which may a a "live" webcast as well as a stored item on a designated server.. In most cases this means that there has to be a trained manager who performs this set up well before the presentation is due. The presenter then connects up anything required and presses a "start" button, delivers the lecture, and presses the "stop" button. There may also be a capability to "see" the capture on a local monitor, and possibly use remote-control cameras and directional microphones if the presentation is highly dynamic.

\section{Camcorders}

The quality and useability of camcorders has advanced in a quite staggering fashion, and with the limited quality (pixel count) required for podcasts these are easily good enough for the purpose.

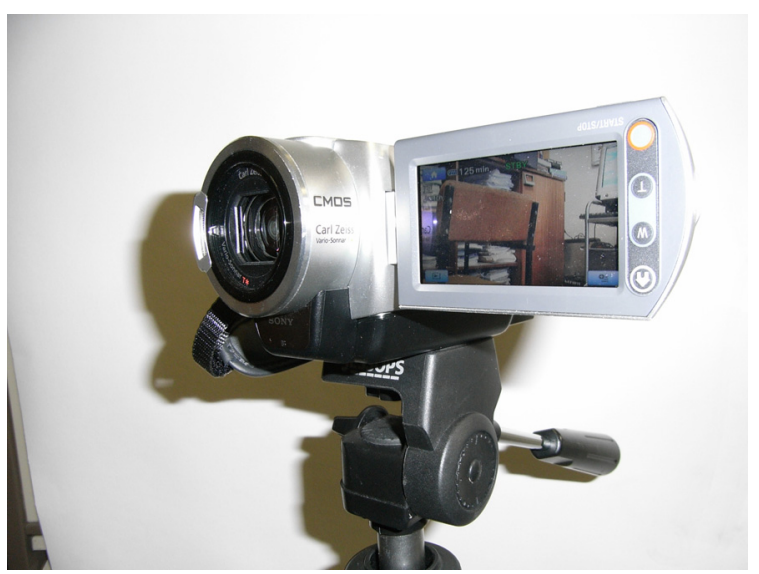

Figure 2. A typical digital camera set up for lecture capture

Tape-based cameras (mini-VHS or Digital-8) are now virtually obsolete and so paradoxically become contenders for widespread use. There are however some difficulties with these older technologies. One basic one is that of battery time - many of these are hungry and so may only last 30 minutes, meaning that a mains supply has to be used constantly. Another is that of capacity - usually one has to choose a lower quality (long-play) to get a full hour of recording. However the most obvious difficulty is that of getting the recorded information into the computer in digital form. Almost all of the more basic cameras have only an analogue video output -usually s-video or composite video - and so have to be played back through a capture device on the computer, taking as long as the recording, and often reducing the quality further. Better quality Digital-8 camcorders may have a DV-OUT port, which allows a "firewire" or IEEE-488 attachment. This obviously requires a PC with a firewire port, and still needs the recording to be played back, albeit with no loss of quality. The top quality camcorders have both DVOUT and DV-IN, which along with the appropriate software gives the PC control over the camcorder operation and allows the video to be extracted directly into a file - however it cannot make the tape go faster so this still takes a long time.
Unfortunately despite their easy availability it is the experience of the authors that tape-based camcorders are unreliable in practice. Constant reuse of the tape soon causes it to become damaged, and this is not usually found until a recovery of a recording is attempted. Storage time of the batteries reduces with use, and the external power connector was rarely designed for permanent use and becomes loose and unreliable. Similarly the video output sockets were not designed for heavy use and the fitment of the cables becomes suspect.

At a greater price point the solution to many of these problems are those camcorders based on hard-disc storage or memory cards. Due to the smaller power drain of "no" moving parts, the battery life is usually over 2 hours. Storage is huge, especially on hard-disc versions where 120 hours at top quality is not uncommon. Memory cards obviously provide as much storage as could be desired, in "lumps" depending on the size of the cards. Experience has shown that a $1 \mathrm{~GB}$ card can hold more than an hour of adequate quality. Direct USB connection also means that getting the files into the computer is simple. The camcorders themselves are also better designed, lighter and easier to use at both capture time and to extract the files. For example the camera shown in Fig 2. above is a hard-disc camera with more than 2 hours of battery life and can hold over 100 hours at top quality - over 4 times the quality required for a computer screen. The screen on the camera can revolve so that as one lectures it is easy to check that one is in the field of view. This particular camera also has a remote control which would allow zooming.. The quality of the image capture is good enough so that normal lighting is fully adequate, so no need for the studio lights the AV techs had to drag around. With such devices being easy to use and very robust, even the smallest department can choose to share one and so it is possible for every lecture of every course to be filmed and stored.

\section{E. Post-Production Issues.}

Many courses already use audio and video segments as part of their teaching - some base their entire unit on such material, delivered from anywhere in the world, such as a unit at the University of Bangor, Wales, delivered by a professor in Cape Town, South Africa (Gibson, 2002). However for entire presentations a common look has evolved - that is a stand-alone window with a small frame "upper-left" with the video of the presenter, a larger frame "upper right" with the presentation, and often smaller frames with stills or text/subtitles towards the bottom. Fig $3 \mathrm{~b}$ shows a typical screenshot.

This section will discuss some ways in which the materials captured can be converted into this format - a task known as post-processing, which as mentioned above took 2 full days for an AV editing technician. There are some dedicated applications for producing "educational resources", which allow a highly interactive mix of features including text, video, audio, quizzes and links to external applications and resources - e.g. Real Presenter or its upgraded replacement PresenterPro (Real, 2008). However these are outwith the scope of this paper, which is concerned with producing a much simpler resource as easily as is possible. 


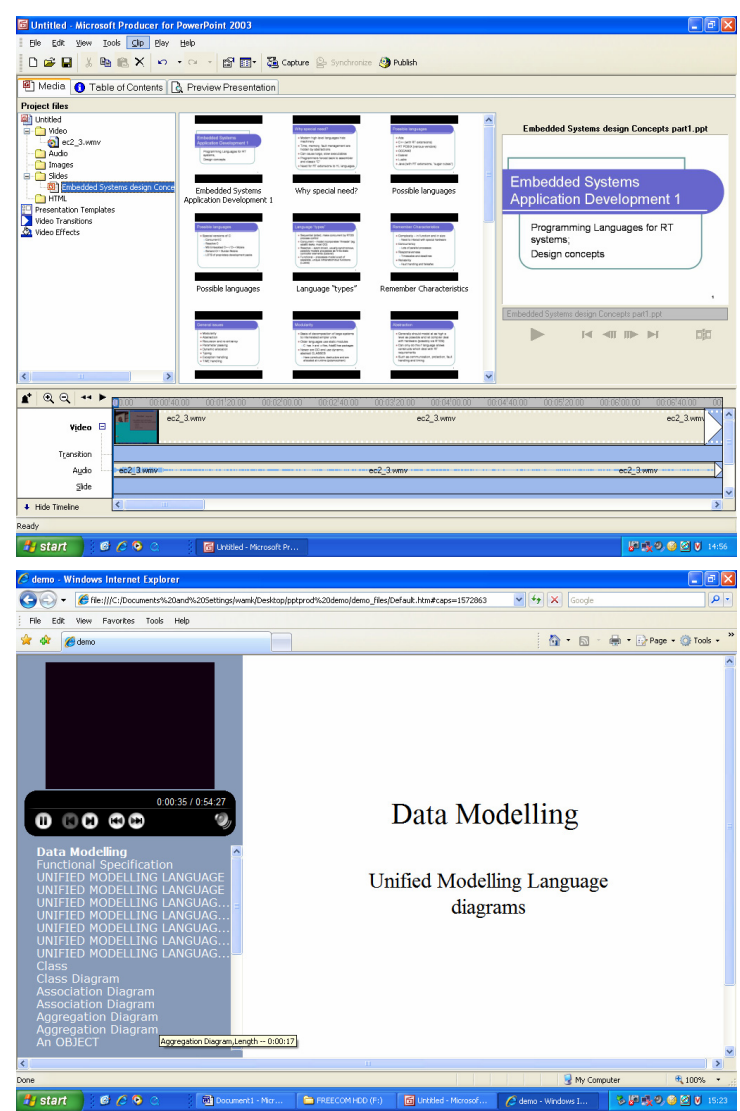

Figure 3a, 3b. Screenshots of Powerpoint Producer and its output

If the presentation is a set of Powerpoint slides, then during the live lecture, a feature called "rehearse timings" is selected rather than the usual "show", which then captures the relative times of the transitions as the slides are advanced. These are stored in the presentation file, and can be seen in the edit view. Then the simplest method is to use a free product (with Microsoft Office) called PowerPoint Producer, as shown in Fig 3a. For each project, a browser is used to pick the presentation, and to place the video file (and the associated sound track - both may be the same file) on the timeline. Then simply choosing "make" gets an output HTML file and assosciated folder which can be viewed on any browser, as seen in the screenshot of Fig $3 b$.

It may be that an HTML set is not an appropriate format - it may be desired to produce a media file which can be viewed as a "movie" or converted to a DVD. There are several ways this can be accommodated, but it must be noted that in most of them the presentation must be saved as a video file rather than a PPT file. If the "capture station" above is being used then this is automatic. In all other cases then there must be a method of converting the PPT file to a video file. The simplest method of doing this is to use a "screen capture" mechanism.

If the "capture station" is available, then it is possible to "loop back" the output video to the capture input. Then simply running the PPT show, using the captured timings, and using the capture application, will provide the movie file.

If a capture station is not available, then there are a number of software-only screen capture applications, such as CamStudio (CamMedia, 2008). Again running the PPT show with this capture application will provide the movie file.

There are several commercial applications which can perform video editing to produce "multiple frame" outputs. For example Premiere (Adobe, 2008) is a fully featured video editing suite which can be configured for "split-screen" operation. However these have the drawbacks of being quite expensive and of having a very steep learning curve.

Note however that producing the movie file from the presentation and then integrating the two files introduces an extra burden on the post-production. For this reason an application was written (LectureShow) to do both at once, using a standard PC without using specialist capture equipment. This application asks for the "lecturer" file (as a video), and the "presentation" file (as either a PPT file or as a video) as shown in Fig 4a. It then starts the CamStudio capture application and runs the two frames simultaneously. The "operator" then only has to watch for the end of the session and hit "exit" for the output file to be produced, ready for storage, as seen in Fig $4 \mathrm{~b}$.
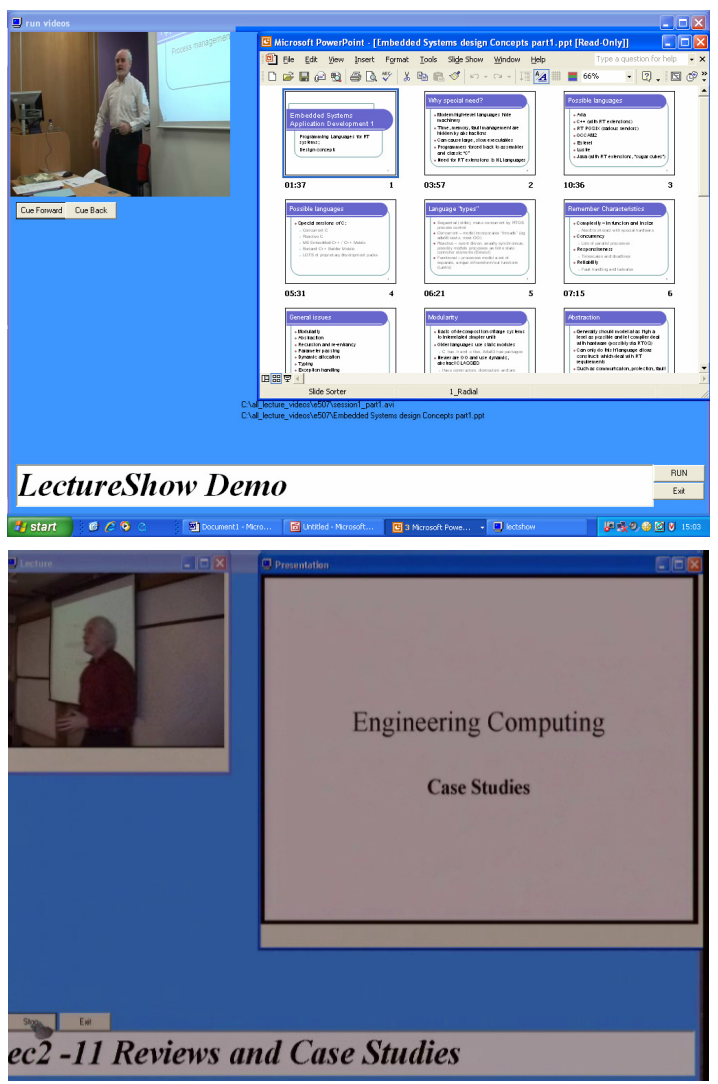

Figure 4a, 4b. Screenshots of LectureShow and its output.

\section{CONCLUSIONS}

The author has now archived a full year of lecture presentations using the various mechanisms described above. This averages 6 hours per week in front of a class, and so the overhead of producing the material had to be low, and given almost no external funding, had to use minimal resources.

It can be clearly stated that the use of the disc-based camcorder and Powerpoint "rehearse" mode made capture trivially easy, while the screen capture application CamStudio along with the self-written display application 
LectureShow allowed a total of only 10 minutes to be spent in post-production, compared with the 2 days of the AV technician.

Was it worth even that amount of effort? The students were asked to record these reactions on the on-line module evaluation mechanism the University hosts:

- Did the recording impact negatively on the performance of the presentation

- Was the quality of the stored lecture sufficient

- Did you use the recording, and was the existence of the recording helpful

- Do you wish this pilot to be extended to other modules

From a total of 230 students, 5 responded.... So the author resorted to direct personal contact with the classes. The anecdotal evidence was overwhelmingly positive. Not a single person could be found who had not watched some of the recordings. None of them had any comment about the quality not being "studio" standard. Interestingly there were a few comments that they lecturer was actually clearer since they were attempting to ensure a good recording with fewer "umms, ahhs and mistakes".

Of special note were a few individuals who stated that they found the recordings indispensable:

- a part-time student who had spent a great deal of the last few months in Poland, who passed the recorded module but failed another;

- a student with long-term illness who kept up with the module from hospital

- a foreign student with poor language skills who stated "I understood you after 3 or 4 listenings"

- one student who said they had "cut" the recordings and attached them to the tutorial questions and solutions.

Several other lecturers were persuaded to join the pilot. None of them had any difficulty in recording the material, and all were satisfied with the files returned to them after post-processing, and did use them in their teaching - one sent copies on $\mathrm{CD}$ to a collaborative course in the middle east. Several have returned to use the system again.

\section{REFERENCES}

[1] Apreso (Now Echo360) http://www.apreso.com/index.asp (accessed April 2008 but superseded)

[2] BlackBoard, "Learning System", http://www.blackboard.com/ us/index.bbb)

[3] Cisco, "Cisco Academy", (accessed April 2008) http://www.cisc o.com/web/learning/netacad/index.html

[4] Echo360 http://www.echo360.com (accessed April 2008)

[5] Gibbs, G, "Preparing To Teach", BPCC Publishing (1992), ISBN $0-947885-56$

[6] Gibson, J "Coastal Law", 2002, (accessed April 2008) http://www.wvn.ac.uk/casestudies/czl/czl.htm

[7] IEE, "Professional development webcasts", http://www.iet.tv/ (accessed April 2008)

[8] Lectopia (Now Echo360) http://lectopia.uwa.edu.au/lectopia/staff/ (accessed April 2008 but superseded)

[9] Philips, R, "Staff and Student perspectives on WBLT" ALT-C (UK), 2007

[10] Real, "PresenterPro", (accessed April 2008) http://www.realne tworks.com/products/presenterpro/index.html

[11] Rose, E, McKee, W, "Workplace Learning", Journal of Learning organisation, 2001, ISSN 0969-6474

[12] Rowntree, D, "Educational development in Curriculum Development", OU press (1992) ISBN 1-85396-040-3

[13] Shulman, L S, and Keislar, E R, "Learning by Discovery - a critical appraisal", Rand McNally (1966)

[14] SUN, "Career Acceleration“, http://www.sun.com/training/c atalog/paths.html (accessed April 2008)

\section{AUTHORS}

W. A. McKee leads the Computer Engineering programme at GCal. (w.a.mckee@gcal.ac.uk).

D. K. Harrison is Director of Research at GCal (dkh@gcal.ac.uk).

M. Allan is Acting Dean of Engineering and Computing at GCal (m.allan@gcal.ac.uk).

Manuscript received 14 October 2008. Published as submitted by the authors. 